\title{
The Pragmatics of Hashtags: Inference and Conversational Style on Twitter.
}

\begin{abstract}
This paper considers the pragmatic contribution of hashtags on the social networking site Twitter. Taking a relevance-theoretic perspective, I argue that hashtags contribute to relevance by adding a layer of activation to certain contextual assumptions and thus guiding the reader's inferential processes. The information contained in a hashtag may guide the hearer in the derivation of both explicitly and implicitly communicated meaning, and may also have stylistic consequences. Twitter facilities one-to-many, asynchronous communication, and so tweeters are unlikely to be able to assume that they share contextual assumptions with all or any of their audience. By allowing tweeters to make their intended contextual assumptions accessible to a wide range of readers, hashtags facilitate the use of an informal, casual style, even in the unpredictable and largely anonymous discourse context of Twitter.
\end{abstract}

\section{Keywords:}

Relevance theory, hashtags, computer-mediated communication, stylistics.

\section{Introduction}

Twitter is an online microblogging site on which users, referred to here as tweeters, can post updates of up to 140 characters in length, known as tweets. These tweets then appear on the feed of anyone who has opted to follow that particular Twitter account, and they can also be found by non-followers via keyword searches using Twitter's in-built search functionality. A prominent feature of Twitter is the use of the \# symbol, which, when followed by a word or phrase, is known as a hashtag 1 . Hashtags function primarily as metadata tags facilitating the retrieval of content from the site. In this article, however, I will argue that the role of

\footnotetext{
1 I will distinguish between the hash symbol itself, and the complete hashtag which comprises both the symbol and the word or phrase that follows it.
} 
hashtags has developed beyond their original purpose, and I will suggest that they now also function to guide readers' interpretations. I will show that they play a stylistic role, allowing users to maintain a personal, informal style in a mediated, largely text-based, public discourse context.

In section 2, I start my discussion by considering the discourse style commonly associated with Twitter, and I suggest that the informal tone is perhaps unexpected given the features of Twitter as a communicative channel. Twitter, as an online platform, facilitates electronically mediated communication. As such, the messages that are posted on the site are constrained by the properties of the interface itself. Most notably, there is a restriction on the length of each tweet. However, other properties of the platform also contribute to the discourse context and constrain users' behaviour. I first consider the stylistic consequences of the Twitter length restriction, and I then use Baym's (2010:7) "seven concepts" of mediated communication to analyse the specific discourse contextual conditions under which interlocutors are communicating when using Twitter.

In section 3, I briefly outline the origins of the hashtag. Then, adopting a relevancetheoretic approach to utterance interpretation (Sperber and Wilson, 1986/95; Blakemore, 1987, 2007; Carston, 2002; Wilson and Sperber, 2004, 2012), I suggest that hashtags have been appropriated by users of Twitter to provide additional contextual information in an economical and stylistically unobtrusive way. This, I suggest, facilitates the production of utterances which are personal, informal and intimate in style in a medium which is public, lean in terms of social cues, and in which the discourse context is dynamic and largely unpredictable.

In section 4, I consider a range of uses of hashtags to illustrate the different ways in which they can be used to guide a reader's inferential processes, and I show that there are attested uses associated with the full range of inferential tasks identified in relevance theory. Finally in section 5 , I sum up the ideas discussed, consider the stylistic role that hashtags fulfil and look to directions for future research in the area. 


\section{Style and Relevance on Twitter}

\subsection{Twitter and conversational style}

According to Twitter, Inc. and at the time of writing, there are 284 million monthly active users on Twitter and 500 million tweets are sent per day, with Twitter supporting over 35 different languages (Twitter, Inc., 2014). Users include celebrities and corporations as well as "ordinary" members (Page, 2011; 2014) and the motivations for tweeting, and the content and style of those tweets can be as diverse as the site membership itself (Marwick and boyd, 2010).

Although broadly a text-based format, Twitter, like other social media platforms combines features from face-to-face spoken interaction with a written language interface (Ferrara, et al., 1991; Georgakapoulou, 1997). Computer-mediated communication more generally has been described as "an Interactive Written Discourse" (Ferrara, et al., 1991:8) and as "neither spoken nor written, yet both" (Baym, 2010: 64). Features more usually associated with faceto-face interaction include its "informality of style" (Georgakapoulou, 1997:142), with Tagg and Seargeant (2014:161) noting that interactions on social media sites "exhibit much of the interactivity and informality that is often found in speech". Zappavigna (2012:127) likewise notes that they "facilitate different kinds of casual, interpersonal interaction", and elsewhere, interactions on Twitter have been described as "conversational exchanges" (Honeycutt and Herring, 2009:1) and "lightweight chat" (Starbird, et al., 2010:242).

Designed as a microblogging site, all updates on Twitter are restricted to 140 characters. It is, therefore, perhaps not surprising that users of the site have developed various techniques for optimising their use of these characters within the "reduced affordances of the character-constrained mode" (Zappavigna, 2012:95). Abbreviations and omissions, of the type previously observed in text messaging (Crystal, 2008; Thurlow and Brown, 2003), are common, and some of these are illustrated in the example in $(1)^{2}$ :

\footnotetext{
2 All of the tweets in this paper were taken from the author's own timeline. They were chosen to illustrate the range of functions that hashtags perform. Usernames and personal names of individuals have been anonymised. The date on which they were originally posted appears after the content of the tweet.
} 
(1) YouGov poll shows 4 the 1st time since the recession that a majority of ppl feel the econ is on the up,but still worried abt cost of living. 30/07/14

Common strategies include the use of numbers or letters to stand for homophonic words or morphemes, as with 4 (for); the omission of non-initial vowels, as with $p p /$ (people) and abt (about); and the shortening of words, as with econ (economy) (Zappavigna, 2012). In example (1), the subject pronoun and copula in the second clause have also been omitted. While in this example, omission of the subject could be grammatical ellipsis driven by considerations of style, the omission of subjects and copula verbs, along with articles and prepositions, is another common strategy used in tweets and restricted registers more generally (Scott, 2010; 2013).

The length restriction and the need to abbreviate and omit elements of the messages mean that the tweeter has to depend on her readers to be able to reconstruct the full intended message from the non-standard, abbreviated forms. According to relevance theory an utterance is no more than a "schematic indication" (Wilson and Sperber, 2012:13) of a speaker's intended meaning. The more attenuated that form is, the more inferential work the reader will have to put in to derive the intended meaning. Furthermore, the character limitation also means that there is often no space to provide contextual information explicitly in the tweet itself. Therefore, the tweeter must also trust that the reader(s) will be able to fill in any background information necessary to interpret the tweet as intended. Construction of a context is also an inferential process (Wilson and Sperber, 2012:13), and so the derivation of the speaker's intended meaning from the tweet alone may carry a heavy inferential, rather than decoding, load (Yus, 2011).

Whenever speakers ${ }^{3}$ produce an utterance, they have the option to make that utterance more or less explicit. Their choices may have stylistic implications. In this article I assume a relevance-theoretic approach to utterance interpretation (Sperber and Wilson, 1986/95; Carston, 2002; Wilson and Sperber, 2004; 2012), and according to Sperber and Wilson:

\footnotetext{
3 While relevance theory usually uses the terms speakerand hearer, the data discussed in this article is mostly written, and so I will also use tweeter and reader. No significant theoretical distinction is intended.
} 
[a] speaker aiming at optimal relevance will leave implicit everything her hearer can be trusted to supply with less effort than would be needed to process an explicit prompt. The more information she leaves implicit, the greater the degree of mutual understanding she makes it manifest that she takes to exist between her and her hearer (1986/95:218).

If a speaker is able to leave aspects of her message implicit and trust that the hearer will still derive the intended interpretation, then she is revealing her confidence in there being a degree of mutual understanding between speaker and hearer. Therefore the character limitation on Twitter contributes to the overall tone of the discourse as informal, casual and conversational (Georgakapoulou, 1997; Starbird, et al., 2010; Zappavigna, 2012; Tagg \& Seargeant, 2014). The speaker assumes a "certain degree of mutuality" (Sperber and Wilson 1986/95:218) between herself and the reader. She reveals that she trusts that the reader will be able to infer much of what she wishes to communicate without the need for overt linguistic prompts.

However, this conversational, casual style might well be regarded as surprising when we consider the type of communication that Twitter facilitates. Markwick and boyd (2010:112) discuss the tension that arises on Twitter from interaction that is both "public-facing and interpersonal". In the next section, I consider Twitter as a communicative channel and discuss its technological and social affordances, exploring a possible basis for this tension. The length restriction on tweets encourages users to leave much of their intended overall message implicit, and yet the nature of Twitter as a communicative channel means they are, in fact, unlikely to be able to assume much, if any, mutuality between themselves and their readers. Once this apparent contradiction has been established, I go on to argue, in section 3 , that the hashtag has been appropriated by users of Twitter as one way to manage the interaction while maintaining the option of using an informal, casual and conversational style.

\subsection{Twitter as a Communicative Channel}

Traditionally, pragmatics is concerned with the interpretation of utterances. As Clark (2013:11) puts it, “[u]tterances are physical entities", which are "written or spoken by a particular person at a particular time and in a particular space". The intended meaning of an utterance can only be derived in context, and, for relevance theory, context is "a 
psychological construct, a subset of the hearer's assumptions about the world" (Sperber and Wilson, 1986/95:15). Part of a hearer's task when interpreting an utterance is to form a hypothesis regarding the speaker's intended contextual assumptions (Wilson and Sperber, 2004). They do this by drawing on information including, but not limited to "information about the immediate physical environment or the immediately preceding utterances", as well as "expectations about the future, scientific hypotheses or religious beliefs, anecdotal memories, general cultural assumptions, [and] beliefs about the mental state of the speaker" (Sperber and Wilson, 1986/95:15-16). However, when communicating online, the cues available to the hearer are much more impoverished than in face-to-face communication (Litt, 2012; Tagg \& Seargeant, 2014), and this will inevitably have an effect on the construction of a hypothesis about the speaker's intended contextual assumptions. Baym (2010) identifies seven features which she argues can be used to compare different media to one another and to face-to-face communication. These are reach, replicability, mobility, temporal structure, storage, interactivity, and social cues. Similarly, boyd (2010) identifies four affordances which she suggests shape users' participation in online communication: persistence, replicability, scalability and searchability. These overlap with and crosscut Baym's seven features. Both prove useful for analysing the discourse context in which communication takes place on Twitter and for understanding how the particular features of the communicative channel affect the strategies that tweeters adopt.

Perhaps the most obviously significant feature of Twitter is its reach potential, or in boyd's terms, its scalability. There is no limit to the number of followers that one account can have, and so a single tweet may appear on millions of Twitter feeds across the world (Marwick \& boyd, 2010; Litt, 2012) ${ }^{4}$. Posts may also easily be replicated when a user reposts a message. This practice, known as "retweeting", reproduces a tweet on the timelines of the retweeter's followers, thus increasing the reach potential even further (boyd, et al., 2010; Marwick and boyd, 2010).

Users may follow particular accounts, but may also search using keywords, and Twitter's searchability also contributes to its reach potential. As Marwick and boyd (2010:99) note, "it

\footnotetext{
${ }^{4}$ At the time of writing the most followed account was @katyperry with $54,810,833$ followers. Source: http://twittercounter.com/pages/100 accessed 29/07/2014.
} 
is virtually impossible for Twitter users to account for their potential audience, let alone actual readers", and while, in reality, most tweets will be read by relatively few people (Marwick and boyd, 2010), their significance lies in the "possibility of tremendous visibility, not the guarantee of it" (boyd, 2010:47). Tweeters are, therefore, writing for an "imagined audience" (Marwick and boyd, 2010; Brake, 2012; Litt, 2012). As boyd (2010:48), points out, "[k]nowing one's audience matters when trying to determine what is socially appropriate to say or what will be understood by those listening". The first of these points has been the focus of much of the existing work on the "imagined audience" in social media (boyd, 2010; Marwick and boyd, 2010; Litt, 2012; Tagg and Seargeant, 2014). Litt (2012:331), for example, focuses on strategies of "self-presentation and impression management", and Marwick and boyd (2010:106) discuss "identity performance" and how users manage "expectations of authenticity" online. Tagg and Seargeant (2014) approach the topic from a social perspective, considering the construction and maintenance of communities online. Both Marwick and boyd (2010) and Wesch (2009) discuss the related notion of "context collapse" in relation to social media. The "potentially limitless" (Marwick and boyd, 2010:97) audience of Twitter "collapse[s] diverse social contexts into one, making it difficult for people to engage in the complex negotiations needed to vary identity presentation, manage impressions and save face" (2010:105).

Equally important, however, and perhaps not so much addressed in the literature, is boyd's second point. The fact that a tweeter is likely to be communicating with an "imagined" or "invisible" (boyd, 2010) audience in a collapsed context will shape the utterance, not only in social terms, but also in terms of how the tweeter ensures her message is understood. Relevance theory strives to explain how utterances are interpreted against the hearer's assumptions about the world. Speakers construct their utterances with the audience and their assumed contextual assumptions in mind, and any "speaker who intends an utterance to be interpreted in a particular way must also expect the hearer to be able to supply a context which allows that interpretation to be recovered" (Sperber and Wilson, 1986/95:16). When posting on Twitter, a user must negotiate a situation in which she is communicating with "an infinitely ambiguous audience in an undefined context" (Wesch, 2009:23), and in which each member of the audience will bring their own set of contextual assumptions to their interpretation of the utterance. She must construct her tweet 
so that her intended meaning is still successfully conveyed despite this collapsed context and the "infinite and ambiguous" (Wesch, 2009:25) nature of the audience and the contextual assumptions that they are likely to hold. The tweeter must strike a balance between including enough information to make the utterance interpretable to as many people as possible, while avoiding the inclusion of easily inferred background information. This must, of course, also be accomplished within the allocated 140 characters.

The availability of specific Twitter applications (apps) on mobile devices means that there is also very little restriction as to where a user might be when they write or read a tweet. That is, Twitter has a high level of mobility. This, in turn, contributes to a flexible and unpredictable temporal structure. A reader may see a tweet a few seconds after it is posted, or they may come across it days, weeks or even years later. If a reader is online at the time of posting, the message may be seen immediately, and when both parties are online, Twitter facilitates synchronous communication. However, tweets remain searchable on the database, and may be accessed at any time after the initial posting, and so, much of the communication on Twitter will be asynchronous. In terms of storage, each user's feed displays a certain number of tweets, and a tweet will be visible on a user's feed until more recent postings displace it. However, all tweets remain on the Twitter database indefinitely, unless the tweet or the host user account is deleted. Tweets, like much online communication, are "persistent-by-default" (boyd, 2010:46). However, as boyd (2010:46) notes, the discourse context does not persist, and "what sticks around may lose its essence when consumed outside of the context in which it was created".

Twitter allows users to be passive observers of online posts and discussions, but it also facilitates interaction. Hashtags, as will be discussed in the next section, were originally intended to link together related tweets. They allowed users to join a conversation on a particular topic and interact with other, perhaps previously unknown, users who were interested in that same topic. Twitter also allows users to send messages to each other, either privately via the direct message function or publically by using the @ tag and the addressee's username (Zappavigna, 2012).

Finally, Twitter is lean in terms of social cues (Litt, 2012). It is mainly text-based, although pictures can be, and often are, added to a post. Interlocutors have no access, however, to facial expressions, gestures, eye contact or prosodic cues. In this respect interaction on 
Twitter patterns more with written language, and users must employ "manipulations of written signs in order to accomplish pragmatic work that would be accomplished by phonological variation, prosody, gaze, posture and other cues in ordinary spoken conversation" (Androutsopoulos, 2013:670). Work by Baron (2009:113) suggests that "paralinguistic cues did not seem to be necessary for clarifying meaning when communicating with friends", but that they take on a more significant role when communicating with strangers. Therefore, in a collapsed context where a tweeter is communicating to an imagined audience, this lack of social cues becomes a significant factor. Tweeters must find means of filling in the gaps left by these communicative cues, or run a higher risk of being misunderstood.

Thus, the discourse context of Twitter, and other similar social media platforms, is a product of each of these properties combining. A user has little or no information about who will be reading her message or at what time they will do so. There is no fixed physical or temporal discourse context, and the tweeter has little or no control over who her audience might be. She must rely on text only to communicate her message, is restricted in the number of characters she may use, and will be unlikely to be able to assume many or any shared contextual assumptions with her readers. This has interesting implications for the strategies that the tweeter might use in her attempt to make her utterance optimally relevant to as many readers as possible. In the next section, I argue that hashtags have developed beyond their original search functionality to allow users to make certain contextual assumptions accessible to their readers, and thus bridge the gap between the tweeter's cognitive environment and the potentially disparate cognitive environments of the readers.

\section{Hashtags and Relevance}

\subsection{Hashtags as search tools and beyond}

Hashtags were not part of Twitter in its original conception. Rather, they grew out of a proposal by Messina (2007) to introduce a system to tag and track content on the site. Originally called "channel tags", these tags were designed so that "not only do we know something specific about that status, but others can eavesdrop on the context of it and then join in the channel and contribute as well" (Messina, 2007). Although hashtags were originally a Twitter-user innovation, in 2009 they were formally adopted as a feature of the 
site (MacArthur, n.d.). The interface was developed so that any string of characters which is preceded by a hash symbol becomes a hyperlink, allowing users to search for any content that includes the same tag. If a large number of people post tweets containing the same hashtag within a short space of time, that hashtag will be said to be trending, and current trends are available for users to browse and view in real time. As Messina points out, an important part of the motivation behind the hashtag was that it would be easy to use without any technical knowledge of coding or search functionality. As he says, hashtags allow users to "track content and updates more relevant and interesting to them without exerting a great deal of extra effort or learning any kind of extraneous of [sic] syntax" (Messina, 2007). Now a fully integrated part of the Twitter interface, hashtags have their own section in the Twitter online support pages, where the following definition is given:

The \# symbol, called a hashtag, is used to mark keywords or topics in a Tweet. It was created organically by Twitter users as a way to categorize messages (Twitter, Inc, n.d.).

The original and most straightforward function of the hashtag is to make the content in the tweets searchable, as in examples (2)-(4).

(2) If you don't want to know what happens in \#Sherlock, avoid spoilers. If you want to know what happens in \#RipperStreet fight for its return 01/01/14

(3) Vienetta Ice \#Failed90sRappers 01/01/14

(4) So it's 2014. A brand new year. How can we make a difference to the care of our patients? Keep your ideas for \#hellomynameis coming... 01/01/14

In example (2) the hashtags \#RipperStreet and \#Sherlock are used to connect tweets relating to particular British television programmes. A hashtag of this type is likely to trend during the airing of the programme, but also remains searchable afterwards. Hashtags may also be associated with internet memes. In (3) the hashtag \#Failed90sRappers links a 
series of joke tweets on the same theme. According to @trendinaliaGB 5 this hashtag trended for 6 hours on the $2^{\text {nd }}$ of January 2014, and is an illustration of an emergent micromeme, which is "both adopted and abandoned in a short period of time" (Huang, et al., 2010:4). On other occasions a hashtag may be designed to coordinate content over a longer timeframe. In example (4), the hashtag \#hellomynameis is used to organise and promote a campaign to encourage better communication from healthcare professionals.

A hashtag may be integrated into the main utterance, as in (2), but it may also be added at the end of a tweet, as in (3). In (4) the hashtag has been adopted as the name of the campaign itself and is used outside of Twitter on general marketing materials.

While connecting, coordinating and promoting content (Yus, 2011; Page, 2012; Zappavigna, 2012) is still the principal function of hashtags, there are many examples of hashtags appearing in tweets where they serve no obvious search function. Consider, for example, the tweet in (5):

(5) I think all drs should be made to lie in a hospital bed wearing PJs \& be stood over. See what it feels like. \#vulnerability \#powerbalance 26/02/14

While the subject of the tweet is the patient-doctor relationship in hospitals, it seems very unlikely that anyone interested in that as a topic would search for it using the hashtags \#vulnerability or \#powerbalance. If they were to do so, they would find many tweets relating to other things alongside the relevant tweet in (5). In short, it would not be an efficient or effective way to access content on that particular subject. The hashtag in this example appears to be performing a function above and beyond the retrieval of content. I argue that in examples such as this, the information in the hashtag is functioning as a guide to the reader's inferential processes when interpreting the utterance. Hashtags of this sort are generally added after the main content of the tweet, as in (5), rather than integrated into the main sentence, as in (2). They are not part of the message proper, but rather provide information about the intended context for interpretation. The inclusion of a hashtag allows the tweeter to make certain contextual assumptions accessible to the reader, without interrupting or disturbing the informal, casual tone of the utterance. They bridge the

\footnotetext{
${ }^{5}$ https://twitter.com/trendinaliaGB
} 
contextual gaps between the tweeter and her potential audience, while maintaining a conversational style within the 140 character limit.

In the next section I briefly introduce the relevance theoretic pragmatic framework, and consider how ideas from relevance theory might help us to understand how hashtags are used on Twitter to raise the accessibility of certain contextual assumptions and thus guide a reader's interpretation.

\subsection{Relevance and Twitter}

According to relevance theory (Sperber and Wilson, 1986/95; Carston, 2002; Wilson \& Sperber, 2004; 2012), human cognition is geared towards maximising relevance. That is, humans are naturally geared towards deriving as many cognitive effects as possible from an input, while expending as little effort as possible. Furthermore, when an input is ostensively addressed to an audience, as is the case with utterances, that audience is entitled to presume that the input will achieve a certain level of relevance for them. They are, in fact, entitled to presume that it will be optimally relevant. An utterance will be optimally relevant if it is (a) "relevant enough for it to be worth the addressee's effort' to process", and (b) "the most relevant one compatible with the communicator's abilities and preferences" (Sperber and Wilson, 1986/95:270). A hearer will look for an overall interpretation which satisfies both of these conditions.

To understand a speaker's overall message, a hearer must perform three sub-tasks, which are typically performed in parallel. First, he must construct a hypothesis about the speaker's intended explicit meaning, that is, he must derive the explicatures of the utterance. According to Sperber and Wilson (1986/95:182), an assumption communicated by an utterance is explicit "if and only if it is a development of a logical form encoded" by the utterance. The basic explicature, also known as the proposition expressed, is derived by

\footnotetext{
${ }^{6}$ According to relevance theory, the crucial type of effort involved in achieving optimal relevance is hearer's processing effort rather than speaker's production effort. See Carston (2005) and Wilson and Wharton (2006) for a discussion of hearer's effort and speaker's effort in relation to relevance theory. The speaker's right to save her own effort is allowed for in clause $b$ of the presumption of optimal relevance, with reference to her "abilities and preferences".
} 
decoding the conceptual content of an utterance, and then enriching that content by disambiguating senses, assigning reference and undertaking various other inferential pragmatic enrichment processes. The basic explicature may itself then be inferentially enriched to yield a series of higher-level explicatures carrying speech-act or attitudinal information (Carston, 2002).

The other two subtasks contribute to the derivation of implicitly communicated meaning. The hearer must construct hypotheses about the intended contextual assumptions (implicated premises) and the intended contextual implications (implicated conclusions). Relevance theory recognises both implicated premises and implicated conclusions as intended implications or implicatures. Consider the exchange in (6):

(6) William: Did you watch X-Factor at the weekend?

Oliver: I don't watch rubbish.

In his reply, Oliver has simply stated that he does not watch rubbish. The basic explicature of his utterance is given in (7).

(7) Explicature: Oliver doesn't watch rubbish (on television).

However, in order for Oliver's reply to answer William's question appropriately, William must access a contextual assumption with which the explicature can combine to yield a relevant contextual implication. In this case, that is the implicated premise in (8). The resulting implicated conclusion is given in (9).

(8) Implicated Premise: X-Factor is rubbish.

(9) Implicated Conclusion: Oliver didn't watch X-factor at the weekend

Although neither is explicitly stated, both (8) and (9) are intentionally communicated, and as such are implicatures.

The outputs of all three tasks (derivation of explicature(s), intended contextual assumptions and intended contextual implications) combine to yield an overall interpretation, which, if all goes well, will satisfy the hearer's expectations of relevance.

Relevance theory treats all forms of verbal communication, whether written or spoken as ostensive. Tweets, just like any other form of ostensive communication, carry with them a 
presumption of their own optimal relevance for their addressees (Yus, 2011). The addresses of an utterance are "the individuals whose cognitive environment the communicator is trying to modify" (Sperber \& Wilson, 1986/95:158). In face-to-face communication, this is likely to be specific individuals, and in one-to-many "broadcast communication", such as Twitter, this will be "whoever finds it relevant" (Sperber and Wilson, 1986/95:158). As Sperber and Wilson (1986/95:158) explain, "the communicator is then communicating her presumption of relevance to whoever is willing to entertain it". As Yus (2011:14) explains, humans are "equipped with a biologically evolved tendency to maximise the relevance of the utterances that we process, but we do not apply different inferential procedures for our interpretation of stimuli (verbal and nonverbal) in physical or virtual contexts". A tweeter, just like any other communicator, will therefore aim for optimal relevance, and will try to make her utterance as relevant as it can be, given her abilities and preferences. The format of Twitter immediately places a constraint on her abilities, as she is limited to using no more than 140 characters in total. Without any physical or prosodic cues available to her (Litt, 2012; Yus, 2011), the tweeter must depend on her limited number of characters to convey her intended message. As discussed in section 2.1, this means that the tweeter is likely to have to leave much of her message implicit, and trust the reader to fill in the gaps inferentially. To do this the reader must identify the tweeter's intended contextual assumptions. Combine this with Twitter as a one-to-many, asynchronous communication channel, and the potential for misunderstanding and miscommunication is high.

However, speakers can lower the risk of miscommunication by making certain contextual assumptions and inferential routes more accessible for the reader. This, I want to suggest, is the key to understanding how tweeters use a format which encourages personal, casual and informal communication to interact with an indefinitely large, time-independent audience. The hashtag, I suggest, has been appropriated to act as a highlighting device. It allows the tweeter to make certain contextual assumptions highly accessible, and thus guides the hearer to the intended overall interpretation in the most efficient and economical manner. 


\subsection{Hashtags as highlighting devices}

When a tweeter adds a hash symbol to a word or phrase on Twitter, thereby creating a hashtag, that tag becomes a hyperlink. A reader clicking on that link will have access to any other tweets which feature the same tag. Therefore, by creating a hashtag, the tweeter is signalling that they consider the tagged word or phrase to be a topic and to be related to, or representative of, the tweet's content. In its original functionality, this enabled a reader to find related content amidst the noise (Page, 2014) of Twitter, and for tags that are integrated into the main message, such as in (2), this remains the main function. However, examples such as (5) (\#vulnerability), suggest that users now treat the hashtag as a means by which they can highlight a topic or theme for the tweet, irrespective of the tag's potential for retrieving related content. The hash symbol, I suggest, has, therefore become a highlighting device. Wilson and Wharton (2006) and Wharton (2009) propose that in face-to-face communication, contrastive stress and pointing or ostensive gazing may be used to "draw attention to a particular constituent in an utterance" (Wharton, 2009:142). Hashtags can be used on Twitter to achieve a similar effect. By highlighting a constituent that might otherwise not have been highlighted, or, indeed, by including an extra, highlighted word at the end of the message, the tweeter is drawing the reader's attention towards that constituent and making it more salient in the reader's cognitive environment. The reader can expect to find relevance in having his attention drawn to a particular constituent in this way, and I suggest that the relevance lies in the extra layer of accessibility that is added to the information encoded by the contents of the tag. By drawing attention to the tagged content, the tweeter can guide the overall interpretation of the utterance by making certain inferential routes more accessible. As discussed in section 3.2, derivation of a speaker's overall intended meaning involves constructing hypotheses about intended explicit content as well as intended implicated premises and conclusions. Inference plays a role in each of these tasks (Carston, 2002), and so we might expect to find hashtags used to guide each of these interpretation processes. In the next section, I discuss examples to illustrate each of these cases. 


\section{Hashtags and Tweeter's Meaning}

\subsection{The proposition expressed}

According to relevance theory, inferential processes contribute not only to what is implicitly communicated by an utterance, but also to what is explicitly communicated (Carston, 2002). Various pragmatic processes are involved in "[c]onstructing an appropriate hypothesis about explicit content" (Wilson and Sperber, 2004:615). These processes include disambiguation, enrichment of vague terms and reference assignment. Yus (2011) discusses the inferential processes that a reader may go through when interpreting a tweet, and notes that they "demand inferential activity similar to the one we apply to the interpretation of other utterances" (Yus, 2011:143). In this subsection, I discuss examples where the content of the hashtag guides the hearer in derivation of the proposition expressed. In the subsections that follow, I turn my attention to the derivation of higher-level explicatures which contribute information about the tweeter's attitude, and then, finally, to implicatures.

According to relevance theory, the inferential processes of disambiguation, saturation (including reference assignment) and free enrichment may all be involved in taking the hearer from the logical form of an utterance to a fully propositional, truth evaluable explicature (Carston, 2002). The tweeter must therefore rely on the reader accessing certain contextual assumptions in order to perform these inferences and, if all goes well, reach the intended interpretation. As discussed in section 2, Twitter, as a communicative channel, is lean in terms of social cues, and provides very little control as to when or by whom the tweet will be read. As a consequence the tweeter is unlikely to be able to predict which contextual assumptions, if any, will be accessible to the, potentially many, readers at the time of processing. However, the inclusion of a hashtag provides the tweeter with a means to raise the accessibility of certain contextual assumptions without including extra content in the main body of the utterance itself. Consider, for example, the tweet in (10):

\section{She's done it! An amazing amazing effort. Please txt FIVE to 70510 \#davina \#windermere}

The tweet in (10) was posted by a charity account during a week in which British television presenter Davina McCall was taking part in a fundraising endurance challenge which included swimming across Lake Windermere. The full challenge lasted a week, and the 
hashtag \#davina allowed Twitter users to follow her progress online. In this respect, the hashtag performed a traditional search function. However, for anyone not actively searching for updates on this topic, the hashtag in (10) may contribute to relevance by raising the accessibility of the intended referent, and so guiding the reference resolution process. To evaluate the truth of the first part of the tweet, repeated here as (11), the reader must infer who she refers to and what it is that she has done.

She's done it!

Followers of the charity's twitter account might well be aware of the ongoing challenge and, so, the intended referent will already be highly accessible to them. Other users will have accessed the tweet after following the \#davina link, and so, again, the intended referent is likely to already be the most accessible one at the point of reading. However, any number of people might come across the message via retweets and promotions, and they could do so days, weeks or months after the tweet was originally posted. The tweeter must aim to make her utterance optimally relevant to as many readers as possible. According to relevance theory (Sperber and Wilson, 1986/95; Wilson and Sperber, 2004), to do this she must construct her utterance so that the first potential referent that the hearer tests is likely to be the intended referent. One way to do this would be to make the utterance more explicit, by tweeting something like (12):

British television presenter Davina McCall has finished her charity swim across Lake Windermere.

While this reduces the inferential processing load required of the reader and lowers the risk of misunderstanding, it requires the reader to process more linguistic information, and it has stylistic implications. As discussed above, the more that is left implicit, the more the speaker indicates that she assumes a mutual understanding. The inverse is also true. Use of a more explicit utterance carries with it a suggestion that shared assumptions cannot be presumed, and so results in a less casual, personal and conversational tone.

The hashtag offers an alternative solution. By including the intended referent in the hashtag, the tweeter is able to make that referent highly accessible. In this example, it performs the role that may be played by a demonstrative gesture in a face-to-face situation 
in which the intended referent is present and can be indicated by, for example, pointing. That is, it adds a layer of activation to a potential referent, making it more likely that the reader will test that referent first. In this example, the hashtag \#davina originally and primarily functions as a search term, connecting all of the tweets relating to the challenge, and many users may have come across the tweet via a hashtag search. However, the fact that it is included, allows the tweeter to assume that Davina will be a highly, if not the most, accessible candidate referent. This frees the tweeter up to use a stylistically preferable pronoun, without risking miscommunication.

While the content of the hashtag performs a dual function in example (10), there are other examples where the hashtag seems to have little or no search functionality, and only contributes to relevance by raising the accessibility of certain contextual assumptions. Consider the example in (13):

Get your fucking snacks and sit the fuck down... \#cinema. 08/02/14

Whereas (10) related to a topical event and was a trending topic on Twitter, (13) has no such contextual connections. It might be hard to imagine why anyone would search using the general term \#cinema, and if they did how the tweet in (13) would be relevant to them. Had the tweeter intended this message to be directed at a specific addressee, we might have expected him to use the "@username" convention to signal this (Zappavigna, 2012). The fact that he has not done this, suggests that the tweet is for more general consumption, and is intended as a comment on behaviour in the cinema that the tweeter finds annoying. The replies that were posted to this tweet suggest that this was the interpretation reached by most readers, as they responded with stories of similarly annoying situations. As with (10), without the information in the hashtag and without any further contextual information, a reader might find it difficult to construct a hypothesis about the intended interpretation of (13), and might eventually abandon the search for relevance. A more explicit version, for example (14), would solve this problem but would, again, have stylistic implications.

(14) People at the cinema (who are disturbing me) should get your fucking snacks and sit the fuck down... 
Part of the expressive force of the utterance in (13) comes from the fact that the tweeter is pretending that he is addressing his fellow cinema-goers directly. The pretend addressees would not require any extra information about the discourse context, and so it would break the pretence to include information about where the speaker is as part of the message itself. Again, the hashtag offers the tweeter an alternative. The noun in the hashtag immediately activates encyclopaedic information associated with a visit to the cinema. Following Wilson and Sperber (2012:181), encyclopaedic entries are likely to contain "ready-made chunks or schemas describing often-encountered sequences of actions or events". Our schemas about a trip to the cinema are likely to include information about buying a ticket, buying some snacks to eat during the movie and then taking your seat. Once this schema has been activated, the reader will hold certain assumptions about the sort of snacks the addressee is likely to be eating and the sort of seat they will be sitting in, and so on. The reader will also assume that you refers to other people in the cinema, or to inconsiderate cinema goers more generally ${ }^{7}$. Furthermore, if the tweeter did not intend the reader to use the cinema schema, then they would have been putting the reader to unnecessary effort by including the hashtag and so encouraging them to activate those associations.

Finally, there are examples where the search functionality of the hashtag may itself facilitate the activation of relevant background assumptions. Consider the tweet in (15):

$$
\text { Sending positive vibes. Positive vibes. Positive vibes. \#mcfc 13/3/14 }
$$

In this example, the main content of the tweet itself is fragmentary and sub-propositional. A reader would be unable to evaluate the truth of the utterance until they have inferred who is sending the positive vibes and who the intended recipient of them is. The tweeter himself is very likely to be the most accessible potential referent for the missing subject pronoun ${ }^{8}$, and so the reader is likely to test that interpretation first. However, without the information in the hashtag, there is no clue as to who the intended object referent might be. The information in the hashtag in this example could potentially contribute to the relevance of the utterance in several ways. For any readers who are familiar with the abbreviation MCFC, Manchester

\footnotetext{
7 This example is interesting from a different perspective, as the actual audience (the tweeter's followers on Twitter) is likely to be different to the purported audience of cinema-goers.

8 See Scott (2013) for further discussion of subjectless sentences in English.
} 
City Football Club will be contextually activated. The reader will then enrich the content of the tweet to derive the proposition expressed in (16).

The tweeter is sending positive vibes to Manchester City Football Club.

For readers who are not familiar with this abbreviation, the search functionality of the hashtag comes into play. By clicking on the hashtag the reader will be taken to a screen displaying other tweets that contain the same tag. This immediately provides the reader with a range of related and continually updated information which he can use to infer the intended meaning of the hashtag, and thus the intended contextual assumptions.

In examples (10), (13) and (15), the information in the hashtags contributes to the derivation of the proposition expressed by the tweets. In the next section, I discuss some examples where the hashtags guide the derivation of higher-level explicatures.

\subsection{Higher-level explicatures}

In the examples discussed above, the information included in the hashtag is needed in order to guide the inferential processes that enrich the logical form to a truth-conditional proposition. However, we find further instances where the content of the hashtag does not seem to have an obvious role in the derivation of the proposition expressed at all. For example, consider (17)Error! Reference source not found.:

$$
\text { One week from today I can start throwing again. \#finally } 01 / 08 / 14
$$

The utterance in (17) is true if and only if the tweeter can start throwing again in one week. However, the hashtag \#finally is part of the ostensively communicated message. It communicates the tweeter's attitude toward the proposition expressed, by revealing that she is relieved by this news and perhaps that she thinks it is overdue. In this example, the information in the hashtag guides the derivation of higher-level explicatures relating to the speaker's attitude. A further example is given in (18), with the proposition expressed given in (19a), and the higher-level explicature in (19b).

(18) Finally got my mom to take and read a @RichardDawkins book while on vacation. \#soproud. 13/3/14 
a. The tweeter finally got her mother to take and read a Richard Dawkins' book while on vacation

b. The tweeter is proud that she finally got her mother to take and read a Richard Dawkins' book while on vacation.

As discussed in section 3.2, in spoken utterances, attitudinal information is often carried by facial expression, gesture, body language or prosodic cues. As none of these is available to the tweeter, the hashtag offers an alternative way in which to convey the speaker's attitude to the proposition expressed.

\subsection{Implicitly communicated meaning}

To understand a speaker's overall message, a hearer must not only work out what the speaker intended to explicitly communicate (explicatures), but also what they intended to implicitly communicate (implicatures). As derivation of implicatures is an inferential process, we might expect to find tweeters using hashtags to guide the implicature derivation process, and this does indeed seem to be the case. Consider example (20), which was posted as a status update on the social networking site Facebook.

I feel like I am falling over on the inside. \#winehangover

In this example, the hashtag does not contribute to the derivation of the proposition expressed. The basic explicature is likely to be something like (21)

The writer feels like she is falling over on the inside.

Neither does the hashtag contribute any speech act or attitudinal information. Rather, it provides background contextual information which guides the overall interpretation of the utterance. Without the hashtag, the relevance of the explicature may not necessarily be clear to the reader, and the message will be open to a wide range of interpretations. It could refer to a physical, emotional or mental falling over, and could be fairly trivial or hugely significant. However, inclusion of the hashtag makes certain contextual assumptions accessible, and the reader is entitled to presume that they will contribute to the overall relevance of the utterance in some way. While the writer has not explicitly stated that she has a wine hangover, the inclusion of the hashtag makes that highly accessible as a 
contextual assumption. Had she not intended the reader to follow this inferential path, then she would have been misleading them by including the hashtag. Once the reader has accessed this implicated premise, given in (22), a range of implicated conclusions become available to him. These will depend on the particular assumptions that the reader holds at the time of reading, but could, for example, include those in (23).

(22) Implicated premise: The writer is suffering from a wine hangover.

(23) Possible implicated conclusions:

(a) The writer's suffering is not serious or life-threatening.

(b) The writer is likely to feel better in a day's time.

(c) The writer does not deserve sympathy as her "falling over" is selfinflicted

Once again, we see that the use of the hashtag has stylistic effects. It allows the writer to avoid including background information as part of the main message itself. Compare (20) with a more explicit alternative in (24):

(24) I drank too much wine last night and have a hangover this morning. I feel like I am falling over on the inside.

The writer of (20) intended to foreground her feelings rather than simply give a description of facts. It is her feelings rather than the cause of them that are relevant. By adding in a description in the alternative in (24), the force of the emotions is reduced as they are no longer the main focus of the utterance.

Compared with the more explicit version in (24), the utterance in (20) also results in more of what Sperber and Wilson call "poetic effects" (Sperber \& Wilson, 1986/95:217; see also Pilkington, 1992; 2000). In (20) the cause, type, and extent of the "falling over" must be inferred on the basis of the hashtag, rather than being explicitly stated. The reader is left largely unconstrained by explicitly communicated content and "is encouraged to be more imaginative and to take a large share of responsibility in imagining" (Sperber and Wilson, 1986/95:221) what it may be like for the writer to be "falling over on the inside". As Sperber and Wilson (1986/95:224) put it, such poetic effects "create common impressions rather than common knowledge". The hearer is encouraged to derive a wide range of weak 
implicatures, rather than one or two strong implications, and this results in the poetic effects which are missing or less effective in the more explicit version.

In example (20), the hashtag functions to make certain contextual assumptions highly accessible and so guide the reader's process of forming a hypothesis concerning the implicated premises. If, as I am arguing, hashtags have been appropriated to guide inferential processes generally, we might also expect to find cases where they guide the formation of implicated conclusions. To illustrate this, consider (25):

Azarenka in shorts. Venus in a dress. \#Contrast. 01/08/14

Again, the hashtag in (25) does not contribute to the propositions expressed by the main body of the tweet. Neither does it express the tweeter's opinion or represent a speech act. Rather, it seems to be instructing the reader to draw a contrast between the two propositions expressed in the tweet itself. That is, it prompts the reader to look for relevance in a contrast between the facts that [Victoria] Azarenka is wearing shorts while Venus [Williams] is wearing a dress. As such it seems to be performing a very similar role to the discourse connectives but and however ${ }^{9}$ (Blakemore, 2002; Iten, 2005; Hall, 2007). It does not contribute to the truth conditions of the utterance, but guides the hearer by indicating that he should look for relevance in a contrast between the two propositions.

The tweet in (26) provides another example of a tweeter using a hashtag to guide the reader in the inferences that he intends him to draw.

Note that no homeopaths representative organisation will take a stand on use of homeopathy for dangerous disease like Ebola. \#complicit 31/7/14

As with examples (20) and (25), the content of the tweet itself is a statement of fact which is true or false in its own right, and the hashtag does not contribute to the truth conditions of

\footnotetext{
9 On the surface, but appears to prompt up to three different interpretations: denial of expectation, contrast and correction. Blakemore (2002) and Iten (2005), amongst others, argue against there being a specific 'contrast' but, and instead proposes that all uses of but activate "an inferential process which results in the contradiction and elimination of an assumption" (Blakemore, 2002:107). While it may be theoretically preferable to have a unitary account, it seems reasonable to assume that a user of twitter would focus on the specific interpretive effects, in this case 'contrast', that were intended on her particular usage.
} 
this proposition. However, the tweeter is clearly directing the reader as to how he should find the proposition expressed relevant. By using the hashtag \#complicit, the reader is prompted to look for who might be complicit and in what. Given the content of the main body of the tweet, it is reasonable to assume that the tweeter intends us to infer that homeopaths who do not comment on the use of homeopathy for dangerous diseases should be held to be complicit in any resulting deaths. Further implicatures might also follow, including, for example, the inference that the tweeter believes that the homeopaths' lack of a stand is irresponsible, and that homeopathy generally should not be supported or promoted. The tweeter has not stated any of these explictly, but the hashtag activates certain assumptions which make them easily accessible as intended implications.

In this section I have provided several examples of hashtags which guide inferential processes which contribute to the implicitly communicated meaning of a tweet. Along with the examples in sections 4.1 and 4.2 , which guide inferential processes relating the explicitly communicated content, we can see that hashtags perform a range of functions relating to all varieties of inference.

\section{Concluding Remarks}

In this article I have taken a closer look at the functions performed by hashtags on Twitter and other social networking sites. I have argued that while their search functionality continues to be important, they have been appropriated by users to perform other roles in the communicative process.

According to relevance theory, inference is involved on both sides of the explicit-implicit divide, and a hearer must carry out a range of inferential work to arrive at the overall intended meaning. This will include deriving the proposition expressed, accessing any higher-level explicatures and deriving any implicatures. In section 4, I have discussed examples relating to each of these levels, and demonstrated that tweeters may use information in hashtags to guide the inferential processes involved in each. This is to be expected on a relevance-theoretic approach to utterance interpretation in which utterances are clues to the speaker's intended meaning, and in which communication is acknowledged to take place at a risk. 
Throughout the discussion I have highlighted the relation between the use of hashtags and the style and tone of the tweets. As discussed in section 2, Twitter facilitates one-to-many, asynchronous communication that is lean in terms of social cues but which remains casual, personal and informal in style. Hashtags provide a means by which tweeters can activate relevant contextual assumptions within the character limit and without the need to provide explicit background information and thus detract from the casual, informal style. In sum, hashtags can be used to activate certain contextual assumptions, thus guiding the reader's inferential processes. This bridges the gap between the tweeter's cognitive environment and the cognitive environments of a potentially disparate range of readers, while allowing a conversational, personal style to be maintained.

The examples discussed in this article have been chosen to illustrate the range of inferential tasks to which hashtags may contribute. Future work in this area is needed to determine the relative frequencies of each function, and therefore to assess to what extent the additional pragmatic role has been incorporated into tweeters' repertoires. Furthermore, we might consider how the inferential function of hashtags fits into a theory of utterance interpretation more generally. Relevance theory recognises the existence of so-called procedural elements (Blakemore, 1987; 2002; 2007) which function as "means for constraining the inferential tasks involved in utterance interpretation" (Blakemore, 2002:89). While procedurally encoded meaning was originally associated with certain functional expressions in language, such as discourse connectives and pronouns, recent work (Wharton, 2009; Wilson, 2011) has suggested that the notion of procedural meaning might be broadened to apply more generally to aspects of communication which "guide the comprehension process in one direction or another" (Wilson, 2011:20). The pragmatic functions of hashtags illustrated in this article seem to fall within this broader definition. Developing a procedural account might be the next step in integrating our understanding of the changing nature of online tagging behaviour into a more general picture of utterance production and interpretation. 


\section{References}

Androutsopoulos, Jannis, 2013. Code-switching in computer-mediated communication. In: Herring, S. C., Virtanen, T. (Eds.) Pragmatics of Computer-Mediated Communication. Mouton de Gruyter, Berlin, pp. 667-694.

Baron, Naomi, 2009. The myth of the impoverished signal: dispelling the spoken-language fallacy for emoticons in online communication. In: Vincent, J., Fortunati, L. (Eds.), Electronic Emotion. Peter Lang, Berlin, pp. 107-136.

Baym, Nancy K., 2010. Personal Connections in the Digital Age. Polity Press, Cambridge. Blakemore, Diane, 1987. Semantic Constraints on Relevance. Blackwell, Oxford.

Blakemore, Diane, 2002. Relevance and Linguistic Meaning: The Semantics and Pragmatics of Discourse Markers. Cambridge University Press, Cambridge.

Blakemore, Diane, 2007. Constraints, concepts and procedural encoding. In: BurtonRoberts, N. (Ed.), Pragmatics. Palgrave MacMillan, Basingstoke, pp. 45-66. boyd, danah, 2010. Social network sites as networked public: affordances, dynamics and implications. In: Papacharissi, Z. (Ed.), Networked Self: Identity, Community and Culture on Social Network Sites. Routledge, Abingdon, pp. 39-58.

boyd, danah, Golder, Scott, Lotan, Gilad, 2010. Tweet, tweet, retweet: conversational aspects of retweeting on Twitter. HICSS-43. IEEE: Kauai, HI, January 6.

Brake, David R., 2012. Who do they think they're talking to? Framings of the audience by social media users. International Journal of Communication 6, 1056-1076.

Carston, Robyn, 2002. Thoughts and Utterances : The Pragmatics of Explicit Communication. Blackwell, Oxford.

Carston, Robyn, 2005. A Note on Pragmatic Principles of Least Effort. UCL Working Papers in Linguistics 17, 271-278.

Clark, Billy, 2013. Relevance Theory. Cambridge University Press, Cambridge. Crystal, David, 2008. Txting: the gr8 db8. Oxford University Press, Oxford.

Ferrara, Kathleen, Brunner, Hans, Whittemore, Greg, 1991. Interactive written discourse as an emergent register. Written Communication 8, 8-34.

Georgakapoulou, Alexandra, 1997. Self-presentation and interactional alliances in e-mail discourse: the style- and code-switches of Greek messages. International Journal of Applied Linguistics 7(2) 141-164. 
Hall, Alison, 2007. Do discourse markers encode concepts or procedures? Lingua 111(1) 149-174.

Honeycutt, Courtenay, Herring, Susan C., 2009. Beyond microblogging: conversation and collaboration in Twitter. HICSS-42. IEEE: Los Alamitos, California, January 5-8.

Huang, Jeff, Thorntonn, Katherine M., Efthimiadis, Efthimis N., 2010. Conversational Tagging In Twitter. Proceedings of the ACM Conference on Hypertext and Hypermedia. Toronto, ON, June 13-16.

Iten, Corinne, 2005. Linguistic Meaning, Truth Conditions and Relevance: the Case of Concessives. Palgrave, Basingstoke.

Litt, Eden, 2012. Knock, Knock. Who's there? The imagined audience. Journal of Broadcasting \& Electronic Media 56(3), 330-345.

MacArthur, Amanda, n.d. The history of hashtags: Shedding some light on the history of hashtags and how we've come to use them. [Online]

Available at: http://twitter.about.com/od/Twitter-Hashtags/a/The-History-Of-Hashtags.htm [Accessed 1210 2014].

Marwick, Alice E., boyd, danah, 2010. I tweet honestly, I tweet passionately: Twitter users, context collapse, and the imagined audience. New Media and Society13, 96-113.

Messina, Chris, 2007. Groups for Twitter; or a proposal for Twitter tag channels. [Online] Available at: http://factoryjoe.com/blog/2007/08/25/groups-for-twitter-or-a-proposal-fortwitter-tag-channels/ [Accessed 730 2014].

Page, Ruth, 2011. Stories and Social Media: Identities and Interaction. Routledge, Abingdon. Page, Ruth, 2012. The linguistics of self-branding and micro-celebrity in Twitter: the role of hashtags. Discourse and Communication 6(2),181-201.

Page, Ruth, 2014. Saying 'sorry': corporate apologies posted on Twitter. Journal of Pragmatics 62, 30-45.

Pilkington, Adrian, 1992. Poetic effects. Lingua 87(1/2), 29-51.

Pilkington, Adrian, 2000. Poetic Effects: a Relevance Theory Perspective. John Benjamins, Amsterdam.

Scott, Kate, 2010. The relevance of referring expressions: the case of diary drop in English. PhD Thesis. University College London. 
Scott, Kate, 2013. Pragmatically motivated null subjects in English: a relevance theory perspective. Journal of Pragmatics 53, 68-83.

Sperber, Dan, Wilson, Deirdre, 1986/95. Relevance: Communiciation and Cognition. Second ed. (with postface) Blackwell, Oxford.

Starbird, Kate, Palen, Leysia, Hughes, Amanda L., Vieweg, Sarah, 2010. Chatter on the red: what hazards threat reveals about the social life of microblogged information. ACM Conference on Computer-Supported Cooperative Work: Savannah, Georgia, February 610, pp. 241-50.

Tagg, Caroline and Seargeant, Philip, 2014. Audience design and language choice in the construction and maintenance of translocal communities on social network sites. In: Seargeant, P., Tagg, C. (Eds.), The Language of Social Media: Identity and Community on the Internet. Palgrace MacMillan, Basingstoke, pp. 161-185.

Thurlow, Crispin, Brown, Alex, 2003. Generation Txt? The sociolinguistics of young people's text-messaging. Discourse Analysis Online 1(1).

Twitter, Inc., 2014. About Twitter, Inc. [Online] Available at: https://about.twitter.com/company [Accessed 212 2014].

Twitter, Inc, n.d. Using hashtags on Twitter. [Online] Available at: https://support.twitter.com/articles/49309-using-hashtags-on-twitter\# [Accessed 730 2014].

Wesch, Michael, 2009. Youtube and you: experiences of self-awareness in the context collapse of the recording webcam. Explorations in Media Ecology 8(2),19-34.

Wharton, Tim, 2009. Pragmatics and Non-Verbal Communication. Cambridge University Press, Cambridge.

Wilson, Deirdre, 2011. The conceptual-procedural distinction: past, present and future. In: Escandell-Vidal, V., Leonetti, M. Ahern, A. (Eds.) Procedural Meaning: Problems and Perspectives. Emerald, Bingley, pp. 3-31.

Wilson, Deirdre, Sperber, Dan, 2004. Relevance theory. In: Horn, L. R., Ward, G. (Eds.) The Handbook of Pragmatics. Blackwell, Oxford, pp. 607-632.

Wilson, Deirdre, Sperber, Dan, 2012. Meaning and Relevance. Cambridge University Press: Cambridge. 
Wilson, Deirdre \& Wharton, Tim, 2006. Relevance and prosody. Journal of Pragmatics 38(10), 1559-1579.

Yus, Francisco, 2011. Cyberpragmatics: Internet-mediated communication in context. John Benjamins, Amsterdam.

Zappavigna, Michele, 2012. Discourse of Twitter and Social Media: How We Use Language to Create Affiliation on the Web. Bloomsbury Academic, London. 